\title{
The Mass of the Galaxy from Large Samples of Field Horizontal-Branch Stars in the SDSS Early Data Release
}

\author{
Timothy C. Beers ${ }^{1}$, Masashi Chiba ${ }^{2}$, Tsuyoshi Sakamoto ${ }^{3}$, Ron \\ Wilhelm $^{4}$, Carlos Allende Prieto ${ }^{5}$, Jesper Sommer-Larsen ${ }^{6}$, Heidi J. \\ Newberg $^{7}$, Brian Yanny ${ }^{8}$, Brian Marsteller ${ }^{1}$, Jeffrey R. Pier ${ }^{9}$ \\ ${ }^{1}$ Dept. of Physics \& Astronomy, Michigan State University, E. Lansing, \\ $M I, U S A$ \\ ${ }^{2}$ Astronomical Institute, Tohoku University, Sendai, Japan \\ ${ }^{3}$ Dept. of Astronomical Science, The Graduate University for Advanced \\ Studies, Mitaka, Japan \\ ${ }^{4}$ Dept. of Physics, Texas Tech University, Lubbock, TX, USA \\ ${ }^{5}$ McDonald Observatory and Dept. of Astronomy, University of Texas, \\ Austin, TX, USA \\ ${ }^{6}$ Theoretical Astrophysics Center, Copenhagen, Denmark \\ ${ }^{7}$ Dept. of Physics $\mathcal{G}$ Astronomy, Rensselaer Polytechnical University, \\ Troy, NY, USA \\ ${ }^{8}$ Fermi National Accelerator Lab, Batavia, IL, USA \\ ${ }^{9}$ US Naval Observatory, Flagstaff Station, Flagstaff, AZ, USA
}

\begin{abstract}
We present a new estimate of the mass of the Milky Way, making use of a large sample of 955 field horizontal-branch (FHB) stars from the Early Data Release of the Sloan Digital Sky Survey. This sample of stars has been classified on the basis of an automated analysis approach, in combination with other methods, in order to obtain estimates of the physical parameters of the stars, i.e., $\mathrm{T}_{\text {eff }}, \log \mathrm{g},[\mathrm{Fe} / \mathrm{H}]$, and should be relatively free of contamination from halo blue stragglers. The stars all have measured radial velocities and photometric distance estimates, and the sample includes objects as distant as $\sim 75 \mathrm{kpc}$ from the Galactic center. Application of a Bayesian likelihood method, for a specific model of the Galaxy, indicates that the total mass of the Galaxy lies in the range $1.5-4.0 \times 10^{12} \mathrm{M}_{\odot}$. Our sample appears to reveal a clear signature of a dual halo population of FHB stars, with the boundary between the inner and outer halo around $20 \mathrm{kpc}$, and the possibility of rather striking differences in the rotational properties of the Galaxy at low metallicity.
\end{abstract}

\section{Introduction}

Kinematic tracers of the distribution of matter in the Galaxy can be used to obtain valuable constraints on both the total mass of the Galaxy and the enclosed mass within certain interesting distances (i.e., within the distance to the Magellanic Clouds, e.g., $r \sim 50 \mathrm{kpc}$ ). In the past, most estimates were based on radial velocities and (where available) proper motions of satellite galaxies of the Milky 
Way, halo globular clusters, and small samples of halo stars on extreme orbits (see Wilkinson \& Evans 1999, and references therein). However, these tracer samples are typically sparsely populated, and often lack accurate measurements of the input quantities, and as a result lead to best estimates of the mass of the Galaxy that are still quite uncertain. In addition, it is well known that such estimates have a rather strong dependence on the inclusion/exclusion of small numbers of very distant objects, such as Leo I, or objects with still-uncertain velocity measures (e.g., Draco and Pal 3).

Recently, Sakamoto, Chiba, \& Beers (2003) obtained new estimates of the mass of the Galaxy based on a large sample of $\sim 400$ relative nearby FHB stars (out to $\sim 10 \mathrm{kpc}$ from the Sun) from the sample of Wilhelm et al. (1999b), including a significant number of objects with available proper motions, in combination with previously analysed satellite galaxies and globular clusters at larger distance. Their mass estimates, although consistent with previous approaches, allow for tighter limits to be placed on the derived mass, in the range $1.5-3.0 \times 10^{12} \mathrm{M}_{\odot}$, with little dependence on the presence of extreme objects such as Leo I. However, this sample of stars draws heavily on tracers chosen from the inner-halo population, which may possess kinematics that are different from the outer halo (see Chiba \& Beers 2000). In order to begin to explore these issues in more detail, herein we analyse a much larger sample of 955 FHB stars, drawn from the Sloan Digital Sky Survey (SDSS; Gunn et al. 1998; York et al. 2000) Early Data Release (EDR), which includes tracers up to some $75 \mathrm{kpc}$ from the Galactic center.

\section{The EDR FHB Stars}

The SDSS EDR (Stoughton et al. 2002) sample includes a total of some 5600 stars with flux calibrated medium-resolution $(R \sim 1800)$ spectra and five-band ugriz photometry over several (non-contiguous) strips on the sky. These data have been used to obtain estimates of stellar physical parameters $\left(T_{\text {eff }}, \log g\right.$, $[\mathrm{Fe} / \mathrm{H}]$ ) based on several methods, including an automated search algorithm (see Allende Prieto et al. 2003), supplemented by independent methods described by Beers et al. (1999) and Wilhelm, Beers, \& Gray (1999a). The accuracy with which we can derive the physical parameters is encouraging, on the order of $\sigma\left(\mathrm{T}_{\text {eff }}, \log \mathrm{g},[\mathrm{Fe} / \mathrm{H}]\right)=(200 \mathrm{~K}, 0.5 \mathrm{dex}, 0.3 \mathrm{dex})$. These methods are particularly useful for the isolation of a "pure" sample of low-surface-gravity FHB stars, distinct from the higher-gravity halo blue stragglers (which exist in approximately equal numbers), and which can confound kinematic analyses if they are not confidently identified and removed from the sample. FHB tracers are particularly useful because reasonably accurate $(\sim 10 \%-20 \%)$ estimates of their distances can be obtained directly from their apparent magnitudes, by adopting well-calibrated estimates of their absolute magnitudes (e.g., Clementini et al. 1995).

Figure 1 shows the derived distances and measured radial velocities for the complete sample of FHB and other A-type stars from the EDR. As can be seen from this figure, the sample of objects includes tracers that are located far out into the halo of the Galaxy, and span a range of radial velocities $-500 \leq V_{\text {los }} \leq$ $+500 \mathrm{~km} / \mathrm{s}$. 

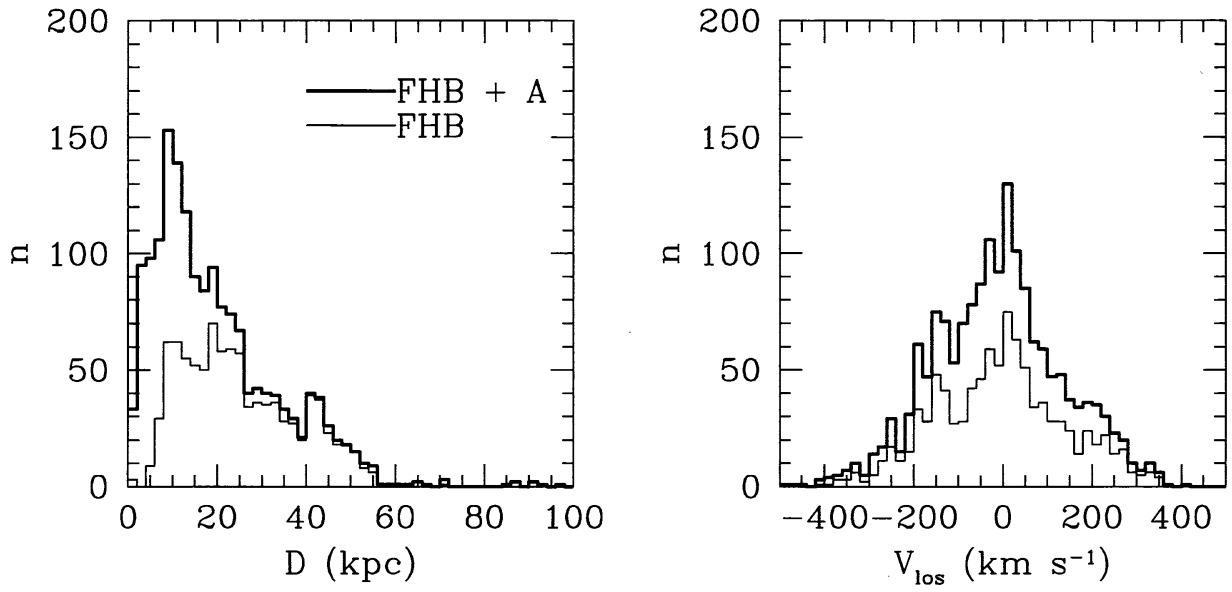

Figure 1. Derived distances, with respect to the Sun, and heliocentric line-of-sight velocities for the EDR sample of FHB (light line) and total sample of FHB + A-type (mostly halo blue stragglers)

\section{Mass Limits from a Bayesian Likelihood Method}

If one models the Galaxy as an isolated, stationary mass distribution, and assumes that all tracer objects are gravitationally bound, then (for a given mass) the rest-frame velocities of the tracers must always be less than the inferred escape velocity at the specified distance of the tracers. Although this method is potentially quite powerful, it is inevitably dependent on small numbers of the most extreme objects with the largest inferred escape velocities. The EDR FHB stars are sufficiently distant that accurate proper motions are not available at present, hence inference of the lower mass limit depends exclusively on the measured radial velocities. For our sample, the derived estimate is not an interesting limit compared to previous analyses.

Therefore, we consider application of an alternative method that takes into account both the positional and kinematic information of all the sample objects, rather than relying on the most extreme velocity tracers. This method, originally suggested by Little \& Tremaine (1987), has been further developed by a number of authors, most recently by Sakamoto et al. (2003), and essentially relies on specific parametric choices for the form of the potential of the Galaxy. This approach introduces a free parameter, $\beta$, that constrains the orbital anisotropy of the tracer population. For simplicity, in this first application, we adopt Model A of Sakamoto et al. (2003), which assumes spherical symmetry and a flat rotation curve in the inner portion of the Galaxy.

We choose to analyse only those data in two of the spatial regions covered by the EDR (which we refer to as regions 1 and 2 below) since the sample drawn from a third region covers only a very small swath of sky. Figure 2 shows the result of our analysis of the EDR FHB stars. The likelihood contours are plotted for three independent samples of FHB stars, separated on the basis of 
their metallicity, and including stars of progressively lower $[\mathrm{Fe} / \mathrm{H}]$. It is perhaps surprising that the inferred mass estimates, although consistent with one another within the estimated errors, appear to depend somewhat on the metallicity cuts chosen. The formal mass estimates we obtain are summarized in Table 1.

Table 1. Derived Masses for Different Cuts in Metallicity

\begin{tabular}{cc}
\hline$[\mathrm{Fe} / \mathrm{H}]$ & Mass $\left(\times 10^{12} \mathrm{M}_{\odot}\right)$ \\
\hline All & 3.9 \\
$<-1.0$ & 3.3 \\
$<-1.5$ & 2.4 \\
$<-2.0$ & 1.5 \\
\hline \hline
\end{tabular}

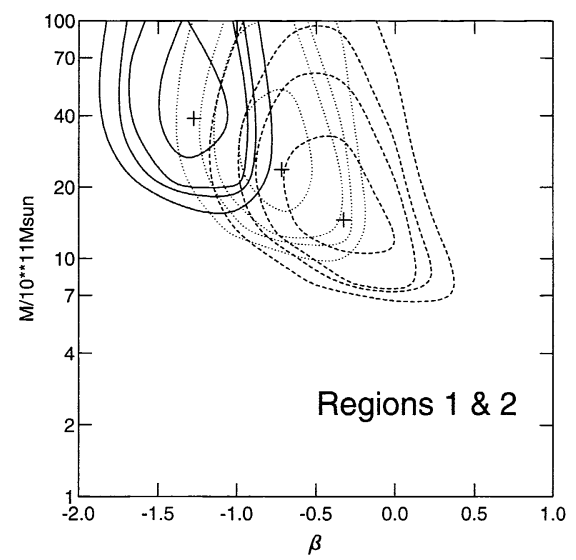

Figure 2. Likelihood contours for mass estimates of the Galaxy, based on a Bayesian analysis, for EDR FHB stars in regions 1 and 2. The crosses locate the estimates associated with the individual metallicity cuts listed in Table 1.

Given the apparent dependence of our derived masses on metallicity, we have undertaken a preliminary investigation of the kinematics of the EDR FHB stars as a function of distance. Although we are limited by the small spatial coverage of the EDR, the inferred rotation $\left(V_{\phi}\right.$, obtained from a Frenk \& White 1980 analysis) of the populations in different metallicity cuts appear quite distinct, and show rather unique signatures for all three metallicity regimes (see Figure 3). The interpretation of this result will clearly have an impact on our derived masses, since one of the primary assumptions is that we are dealing with a non-rotating halo population. 

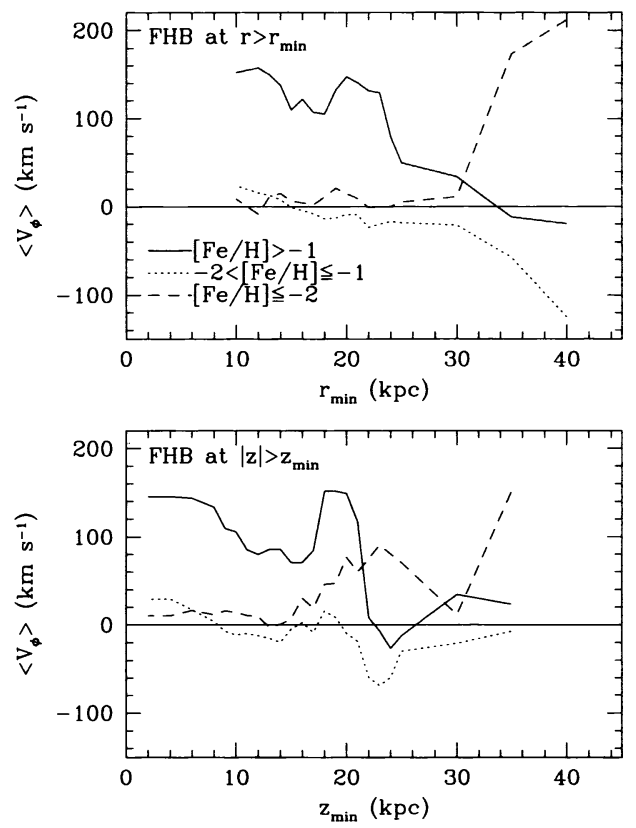

Figure 3. Inferred rotation for various metallicity cuts of the EDR FHB sample. Note the apparently strong change in kinematics for distances outside $r_{\min }$ and $z_{\min }>20 \mathrm{kpc}$, where $r_{\min }$ is the minimum Galactocentric distance included in the cut and $z_{\min }$ is the minimum distance above and below the Galactic plane included in the cut.

\section{Future Work}

The first public data release (DR1) from SDSS is now available (Abazajian et al. 2003), and we are in the process of expanding our analysis to include the $\sim 3000$ FHB stars we are able to isolate from these data. Future samples of FHB stars from SDSS will enable analyses of several tens of thousands of tracer objects, with an expected dramatic refinement in our mass estimates. Clearly, further inspection of the surprising inferred kinematics of the FHB stars will be required, and if the signatures we have noted in the EDR sample remain, we will have to modify the methodology used to include the presence of rotating populations of tracers. Indeed, further searches for distinct kinematics of lowmetallicity stars at large distances from the Galactic center are important for several reasons, including the possibility that they arise from debris of recognized or new streams from captured satellites (e.g., Newberg et al. 2003).

Acknowledgments. T.C.B. acknowledges partial support from NSF grants AST 00-98508 and AST 00-98549. H.N. acknowledges partial support from The Research Corporation and NSF grant AST 03-07571. Funding for the creation and distribution of the SDSS Archive has been provided by the Alfred P. Sloan 
Foundation, the Participating Institutions, the National Aeronautics and Space Administration, the National Science Foundation, the U.S. Department of Energy, the Japanese Monbukagakusho, and the Max Planck Society. The SDSS Web site is http://www.sdss.org/.

\section{References}

Abazajian, K. et al. 2003, AJ, 126, 2081

Allende Prieto, C., Beers, T.C., Li, Y., Newberg, H.J., Wilhelm, R., \& Yanny, B. 2003, in Carnegie Observatories Astrophysics Series, Vol. 4, Origin and Evolution of the Elements, eds. A. McWilliam and M. Rauch (Pasadena: Carnegie Observatories), astro-ph/0304352

Beers, T.C., Rossi, S., Norris, J.E., Ryan, S.G., \& Shefler, T. 1999, AJ, 117, 981 Chiba, M., \& Beers, T.C. 2000, ApJ, 119, 2843

Clementini, G., Carretta, E., Gratton, R., Merighi, R., Mould, J., R., \& McCarthy, J.K. 1995, AJ, 110, 2319

Frenk, C., \& White, S. D. M. 1980, MNRAS, 193, 295

Gunn, J.E., et al. 1998, AJ, 116, 3040

Little, B. \& Tremaine, S. 1987, ApJ, 320, 493

Newberg, H.J., et al. 2003, ApJ, 596, L191

Sakamoto, T., Chiba, M., \& Beers, T.C. 2003, A\&A, 397, 899

Stoughton, C. et al. 2002, AJ, 123, 485

Wilhelm, R. Beers, T.C., \& Gray, R.O. 1999a, AJ, 117, 2308

Wilhelm, R., Beers, T.C., Sommer-Larsen, J., Pier, J.R., Layden, A.C., Flynn, C., Rossi, S., \& Christensen, P.R. 1999b, AJ, 117, 2329

Wilkinson, M.I. \& Evans, N.W. 1999, MNRAS, 310, 645

York, D.G. et al. 2000, AJ, 120, 1579 